\title{
Diagnostic Yield of High-resolution Esophageal Manometry With Chicago Classification Version 3.0 in Thai Patients
}

\author{
Sawangpong Jandee ${ }^{1,2 *}$ and Kasemsak Jandee ${ }^{3,4}$ \\ ${ }^{I}$ Gastroenterology and Hepatology Unit, Division of Internal Medicine, Faculty of Medicine, Prince of Songkla University, Hat Yai, Songkhla, \\ Thailand; ${ }^{2}$ Nanthana-Kriangkrai Chotiwattanaphan Institute of Gastroenterology and Hepatology, Songklanagarind Hospital, Hat Yai, Songkhla, \\ Thailand; ${ }^{3}$ School of Public Health, Walailak University, Tha Sala, Nakhon Si Thammarat, Thailand; and ${ }^{4}$ Epidemiology Unit, Faculty of \\ Medicine, Prince of Songkla University, Hat Yai, Songkhla, Thailand
}

\section{Background/Aims}

High-resolution manometry with the Chicago classification scheme has been introduced in clinical practice as a gold standard for esophageal motility test. This study aims to evaluate the diagnostic yield of high-resolution manometry in Thai patients.

\section{Methods}

All available high-resolution esophageal manometry (HREM) studies performed during the study period were retrospectively reviewed and interpreted according to the Chicago classification version 3.0. The main esophageal symptoms and coexisting factors were correlated with the HREM findings.

\section{Results}

Of the 201 patients, nearly half (49.8\%) were documented to have dysphagia. The second most common condition was refractory reflux symptoms (17.4\%). More than $70.0 \%$ of dysphagia patients showed abnormal esophageal motility, contrary to globus patients who mostly had normal test findings (65.4\%). Dysphagia still was the most often correlated condition with major esophageal motility disorders (88.7\%), particularly the elderly patients who have coexisting weight loss. Endoscopic and/or surgical procedures were revealed for the highest rate among patients with dysphagia but no one in the globus group needed this intervention. The sensitivity and specificity of dysphagia for major esophageal motility disorders were $70.0 \%$ and $67.0 \%$. A much lower sensitivity and higher specificity were found in other non-dysphagia symptoms, especially nausea/vomiting or belching (3.0\% or $89.0 \%)$. The highest positive likelihood ratio (2.10) to detect major abnormalities was also observed in dysphagia.

\section{Conclusion}

Esophageal manometry provided the highest yield in dysphagia; it was not a strongly beneficial test in patients presenting with nondysphagia to identify clinically relevant esophageal motor disorders.

(J Neurogastroenterol Motil 2021;27:533-539)

\section{Key Words}

Deglutition disorders; Diagnosis; Esophageal motility disorders; Esophagus; Manometry

Received: April 24, 2020 Revised: March 28, 2021 Accepted: April 23, 2021

(.) This is an Open Access article distributed under the terms of the Creative Commons Attribution Non-Commercial License (http://creativecommons. org/licenses/by-nc/4.0) which permits unrestricted non-commercial use, distribution, and reproduction in any medium, provided the original work is properly cited.

*Correspondence: Sawangpong Jandee, MD

Gastroenterology and Hepatology Unit, Division of Internal Medicine, Faculty of Medicine, Prince of Songkla University, 15 Karnjanavanich Road, Hat Yai, Songkhla 90110, Thailand

Tel: +66-0-7445-1451, Fax: +66-0-7442-9385, E-mail: tekikung@gmail.com, sawangpong.j@psu.ac.th 


\section{Introduction}

Esophageal manometry is an important and recommended test to evaluate esophageal motility, which is indicated in the diagnostic evaluation of patients with non-obstructive dysphagia and before anti-reflux surgery to assess the adequacy of peristalsis. ${ }^{1}$ Manometry is also used in other clinical settings such as non-cardiac chest pain (NCCP) and regurgitation. ${ }^{2,3}$

High-resolution manometry (HRM), providing enhanced pressure resolution and standardized metrics has been introduced into research and clinical practice to accurately diagnose esophageal motility disorders. ${ }^{4,5}$ Although the computer-generated automated analysis of a HRM study is a useful aid, the final interpretation needs to be performed by an experienced and competent clinician. A benchmark for competency in HRM interpretation has been suggested the experience of interpreting 50 studies $^{6}$ and requires some degree of hands-on training in a center of excellence.

The Chicago classification version 3.0 (v3.0) is applied to determine the manometric diagnosis to disorders with esophagogastric junction (EGJ) outflow obstruction as well as major and minor disorders of peristalsis. ${ }^{7}$ Achalasia and major disorders of peristalsis have corresponding managements, contrary to minor esophageal motility disorders for which there is still no established treatment.

Prospective and consecutive data from an Indian study in $2017,{ }^{8,9}$ suggested that dysphagia was the only symptom to have a high likelihood ratio and positive predictive value to pick up esophageal motility disorders, while the other symptoms did not have a clear benefit for manometry testing and needed to be studied further. The weak points of this study are its small sample size, the utilization of different HRM machines from those in the original literature, and the correlations did not pay attention to the specific subgroup of major esophageal motility disorders, which have much more impact in clinical practice.

In Thailand, we lack specialists in this field, including those that are responsible for esophageal motility testing with HRM. Moreover, we still do not have data related to HRM in terms of the predictive ability of esophageal symptoms or the indication for testing concerning major HRM findings. This would help physicians in their decision-making to apply HRM testing with a high cost-effectiveness in Thailand and other limited-resource countries, especially in referral centers. This study aims to assess the predictive ability of esophageal symptoms for major esophageal motility disorders in Thai patients using high-resolution esophageal manometry (HREM) according to the Chicago classification v3.0.

\section{Materials and Methods}

All available adult HREM findings generated with a 36-channel solid-state catheter system with circumferential sensors $1 \mathrm{~cm}$ apart, Sierra HRM assembly (Given Imaging, Los Angeles, CA, USA) at Songklanagarind Hospital, a large, single, tertiary-care and referral center of esophageal motility disorders in the South of Thailand, from April 2017 (1st available HRM) to December 2019 were retrospectively reviewed and interpreted according to the Chicago classification v3.0 by a gastroenterologist experienced in HREM interpretation (more than 50 tracing interpretations as a benchmark of competency). All HREM studies were performed after 6 hours of fasting. All subjects completed the 10 test swallows protocol ( $5 \mathrm{~mL}$ ambient-temperature water swallows at least $20 \mathrm{sec}-$ onds apart) in the semi-upright or supine position. We consecutively enrolled all eligible HREM tracings and excluded incomplete tracings that could affect the interpretation.

According to Chicago classification v3.0, we classified the HREM findings into either a specific diagnosis (achalasia, EGJ outflow obstruction, absent contractility, distal esophageal spasm (DES), hypercontractile esophagus, ineffective esophageal motility, fragmented peristalsis, and normal finding) or a major diagnosis (disorder of EGJ outflow obstruction, major disorders of peristalsis, minor disorders of peristalsis, and normal finding).

The main esophageal symptoms or indications for esophageal manometry were categorized into dysphagia, non-cardiac chest pain, refractory reflux symptoms, globus, and nausea/vomiting or belching. The stepwise diagnostic algorithm according to the main esophageal symptom is shown in Figure 1. All patients had

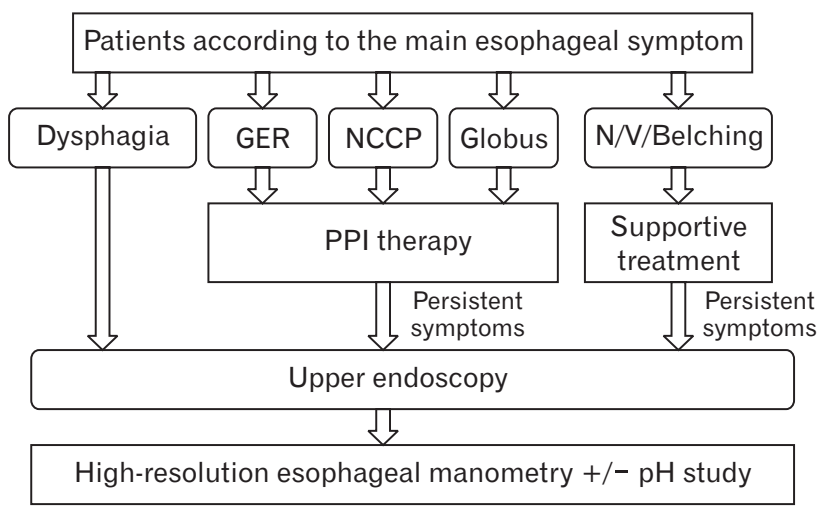

Figure 1. Stepwise diagnostic algorithm of patients according to main esophageal symptoms. GER, gastroesophageal reflux; NCCP, noncardiac chest pain; N/V, nausea/vomiting; PPI, proton pump inhibitor. 
undergone upper endoscopy before the HREMs were performed. Moreover, the managements after esophageal manometric findings as well as balloon dilatation, botulinum toxin injection, medications or surgery were identified. The patients' demographic data including age and sex was also collected.

Based on a previous study, ${ }^{8}$ the prevalence of esophageal motility disorders among HREM testing patients is $65.0 \%$, and the sensitivity of dysphagia symptoms to indicate esophageal motility disorders is $58.0 \%$. Thus, at least 145 HERM tracings were needed by estimating a single proportion sample size calculation for a $10 \%$ margin of error and a $95 \%$ confidence interval. This study was approved by the Office of Human Research Ethics Committee, Faculty of Medicine, Prince of Songkla University (REC. $62-$ 439-14-1).

\section{Data Access and Ethics}

The data of all patients undergoing esophageal HRM at Songklanagarind Hospital from 2017 until December 2019 were collected. The primary investigator, who was also the main interpreter, accessed the baseline data and HRM tracing findings. The patients' data were coded. The research investigators were obligated to protect the data from disclosure outside the research according to the terms of the research protocol. The subjects' names or other identifiers were stored separately from their research data.

\section{Study Outcome}

\section{Primary outcome}

This study aims to evaluate predictive ability of dysphagia symptom for major esophageal motility disorders on HREM findings according to the Chicago classification v3.0 (disorders of EGJ outflow obstruction or major disorders of peristalsis) using sensitivity, specificity, positive and negative likelihood ratios, and positive and negative predictive values.

\section{Secondary outcome}

We would like to determine predictive ability of other nondysphagia symptoms for major esophageal motility disorders on HREM findings according to the Chicago classification v3.0. Prevalence of each HREM finding according to esophageal symptoms or indication for esophageal manometry testing were identified. Patients' managements according to esophageal symptoms and HREM findings were also evaluated.

\section{Statistical Methods}

The categorical data are shown using percentages. The predictive ability of esophageal symptoms for major esophageal motility disorders were assessed by calculating the sensitivity, specificity, positive and negative likelihood ratios, and positive and negative predictive values with a $95 \% \mathrm{CI}$. The associations of demographic characteristics, coexisting symptoms, and the detection of major esophageal motility disorders were subgroup analyzed in patients with dysphagia using multiple logistic regression. Data were analyzed by R version 3.5.3 (R Foundation for Statistical Computing, Vienna, Austria, 2019).

\section{Terms and Definitions}

We mainly classified abnormal esophageal motility tests into major and minor esophageal motility disorders. The major esophageal motility disorders were disorders with EGJ outflow obstruction (achalasia and EGJ outflow obstruction) and major disorders of peristalsis (DES, jackhammer esophagus, and absent contractility). Meanwhile, the minor esophageal motility disorders were minor motility disorders (ineffective esophageal motility and fragmented peristalsis).

Table 1. High-resolution Esophageal Manometry Results According to Esophageal Symptoms

\begin{tabular}{|c|c|c|c|c|c|c|}
\hline HREM findings & $\begin{array}{c}\text { Dysphagia } \\
(100[49.8])\end{array}$ & $\begin{array}{c}\text { GERD } \\
(35[17.4])\end{array}$ & $\begin{array}{c}\mathrm{NCCP} \\
(25[12.4])\end{array}$ & $\begin{array}{c}\text { N/V/Belching } \\
(15[7.5])\end{array}$ & $\begin{array}{c}\text { Globus } \\
(26[12.9])\end{array}$ & $\begin{array}{c}\text { Total } \\
(201[100])\end{array}$ \\
\hline Normal & $29(29.0)$ & $20(57.1)$ & $11(44.0)$ & $6(40.0)$ & $17(65.4)$ & $83(41.3)$ \\
\hline Abnormal & $71(71.0)$ & $15(42.9)$ & $14(56.0)$ & $9(60.0)$ & $9(34.6)$ & $118(58.7)$ \\
\hline Major esophageal motility disorders & $63(88.7)$ & $8(53.3)$ & $10(71.4)$ & $3(33.3)$ & $6(66.7)$ & $90(76.3)$ \\
\hline Disorders with EGJ outflow obstruction & $46(73.0)$ & $5(62.5)$ & $5(50.0)$ & $1(33.3)$ & $5(83.3)$ & $62(52.5)$ \\
\hline Major disorders of peristalsis & $17(27.0)$ & $3(37.5)$ & $5(50.0)$ & $2(66.7)$ & $1(16.7)$ & $28(23.7)$ \\
\hline Minor disorder of peristalsis & $8(11.3)$ & $7(46.7)$ & $4(28.6)$ & $6(66.7)$ & $3(33.3)$ & $28(23.7)$ \\
\hline
\end{tabular}

HREM, high-resolution esophageal manometry; GERD, gastroesophageal reflux disease; NCCP, non-cardiac chest pain; N/V, nausea/vomiting; EGJ, esophagogastric junction.

Data are presented as $n(\%)$. 


\section{Results}

Two hundred and twenty-eight patients underwent HREM during the study period. Two hundred and one patients were included based on the completeness of data and HRM tracings criteria. Most patients were female (58.2\%), and the median (interquartile range) age was $56(42,65)$ years. Nearly half of the patients (49.8\%) underwent esophageal manometry due to dysphagia problems. The second most common indication were refractory reflux symptoms (17.4\%). We noticed an equally small proportion of patients with either NCCP or globus were sent for manometric testing. The lowest percentage of those undergoing the test belonged to patients with nausea/vomiting or belching (7.5\%; Table 1).

\section{High-resolution Esophageal Manometry Results in Correlation With Esophageal Symptoms}

The highest yield of detecting esophageal motility disorders, accounting for more than $70.0 \%$, was observed among patients with dysphagia. This was in contrast to patients with globus, who mostly had normal motility test findings (65.4\%). When we considered the abnormal esophageal motility findings, dysphagia was still the most common symptom that correlated with major esophageal motility disorders (88.7\%) followed by NCCP (71.4\%), globus (66.7\%), GERD (53.3\%), and nausea/vomiting or belching (33.3\%) (Table 1).

For dysphagia, the most common major esophageal abnormality among these patients was achalasia 35/63 (55.6\%) followed by EGJ outflow obstruction 11/63 (17.5\%) and absent contractility 9/63 (14.3\%). Meanwhile, EGJ outflow obstruction (4/10, 40.0\%), and jackhammer esophagus $(4 / 10,40.0 \%)$ were common in
NCCP. Among GERD patients, we notably picked up some major esophageal motility disorders consisting of EGJ outflow obstruction (4/8, 50\%), jackhammer esophagus (2/8, 25.0\%), and achalasia and DES in equal proportions $(1 / 8,12.5 \%)$. In spite of the fact that the lowest yield of detecting abnormal esophageal motility disorders was observed among globus patients (34.6\%), EGJ outflow obstruction was found to be a major esophageal motility disorder $(5 / 6,83.3 \%)$. Among patients with an abnormal esophageal motility test, nausea/ vomiting or belching was predominantly related with minor esophageal disorders $(6 / 9,66.7 \%)$; however, major esophageal motility disorders such as achalasia and DES may also be present (Fig. 2).

EGJ outflow obstruction was found in all symptoms except for patients with nausea/vomiting or belching. Similarly, DES also was detected in a variety of symptoms, but it was not found in NCCP patients. Finally, minor disorders of peristalsis were found among all symptoms, but they were the least correlated with dysphagia $(11.3 \%)$.

\section{Post-manometry Treatments in Correlation With Esophageal Symptoms}

Of the study population, 153/201 (76.1\%) patients received some medications as treatment of choice after esophageal manometry was performed. The highest endoscopic or surgical rate was observed in patients with dysphagia (42.0\%) followed by NCCP (12.0\%), nausea/vomiting or belching (6.7\%), and GERD (5.7\%). However, no patient in the globus group needed this procedure.

\section{Predictive Ability of Esophageal Symptoms for Major Esophageal Motility Disorders}

The sensitivity and specificity of dysphagia for major esopha-

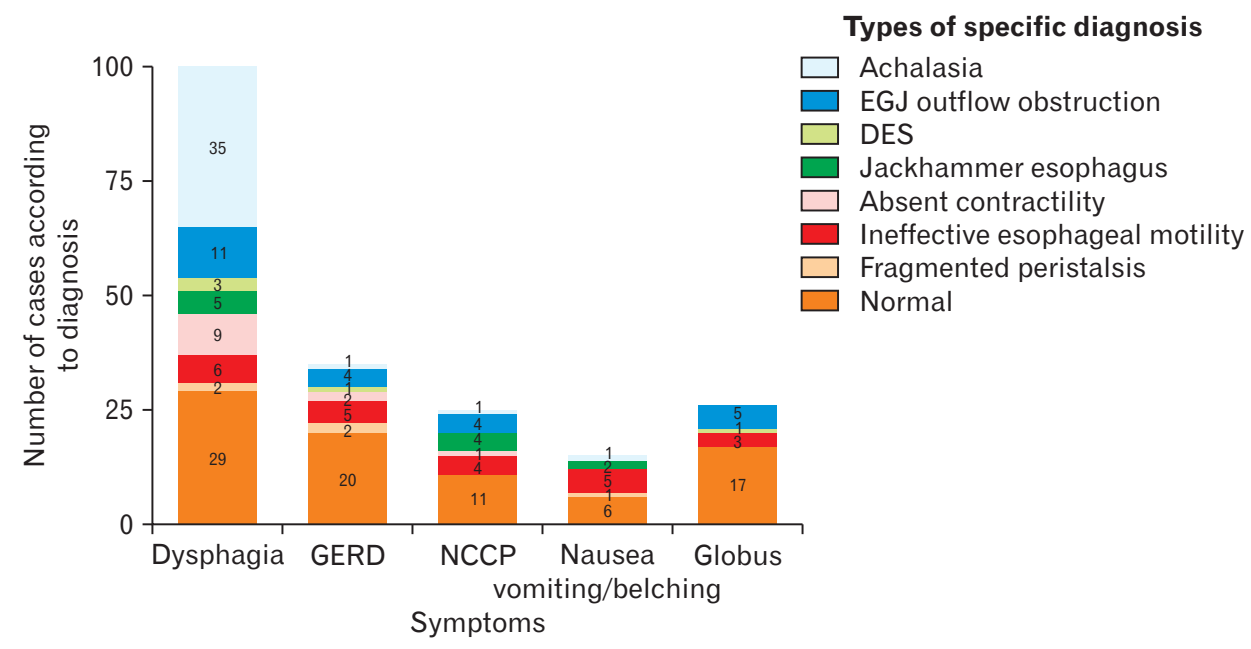

Figure 2. Specific diagnosis of esophageal manometry according to main esophageal symptoms. GERD, gastroesophageal reflux disease; NCCP, noncardiac chest pain; EGJ, esophagogastric junction; DES, distal esophageal spasm. 
geal motility disorders were $70.0 \%$ and $67.0 \%$, respectively. Meanwhile, a much lower sensitivity and higher specificity were observed in other non-dysphagia symptoms, especially nausea/vomiting or belching (sensitivity $3.0 \%$ and specificity $89.0 \%$ ) (Table 2 ). The positive and negative predictive values of dysphagia were $63.0 \%$ and $73.0 \%$, respectively. This was in contrast to nausea/vomiting or belching, which demonstrated the lowest positive predictive value. The highest positive likelihood ratio (2.10) to detect major esophageal motility disorders was found in dysphagia symptoms.

\section{Factors Associated With Detection of Major Esophageal Motility Disorders Among Patients With Dysphagia}

Regarding to the results, dysphagia provided the highest yield for major esophageal motility disorders. We subsequently analyzed to find out the factors associated with these abnormalities using multiple logistic regression (Table 3). Patients with dysphagia who have coexisting weight loss were more likely to be diagnosed with major esophageal motility disorders with an adjusted odds ratio of 7.11 (95\% CI, 1.05-21.00). In addition, the elderly patients ( $\geq 65$ years) were more likely than the younger groups to detect major esophageal motility disorders.

\section{Discussion}

It is widely accepted that esophageal manometry is the gold standard investigation for assessing esophageal motility. Furthermore, high-resolution manometry following the Chicago classification have become widely adopted into clinical practice. The recommended indications for esophageal manometry testing are confined to patients with non-obstructive dysphagia and before anti-reflux surgery, but this investigation has also been applied to other scenarios such as NCCP and regurgitation. A point of concern is its applicability in Thailand and Southeast Asian countries, which still lack

Table 2. Sensitivity, Specificity, Positive Predictive Values, and Negative Predictive Values of Symptomatic Variables for Major Esophageal Motility Disorders

\begin{tabular}{lccccrc}
\hline \multicolumn{1}{c}{$\begin{array}{c}\text { Symptomatic } \\
\text { variables }\end{array}$} & $\begin{array}{c}\text { Sensitivity } \\
(95 \% \mathrm{CI})\end{array}$ & $\begin{array}{c}\text { Specificity } \\
(95 \% \mathrm{CI})\end{array}$ & $\begin{array}{c}\text { PPV } \\
(95 \% \mathrm{CI})\end{array}$ & $\begin{array}{c}\text { NPV } \\
(95 \% \mathrm{CI})\end{array}$ & $\begin{array}{c}\text { LR+ } \\
(95 \% \mathrm{CI})\end{array}$ & $\begin{array}{c}\text { LR- } \\
(95 \% \mathrm{CI})\end{array}$ \\
\hline $\begin{array}{l}\text { Dysphagia } \\
\text { GERD }\end{array}$ & $0.70(0.59,0.79)$ & $0.67(0.57,0.75)$ & $0.63(0.53,0.72)$ & $0.73(0.64,0.82)$ & $2.10(1.56,2.82)$ & $0.45(0.32,0.63)$ \\
NCCP & $0.09(0.04,0.17)$ & $0.76(0.67,0.83)$ & $0.23(0.10,0.40)$ & $0.51(0.43,0.58)$ & $0.37(0.17,0.76)$ & $1.20(1.06,1.36)$ \\
Nausea, vomiting, \\
$\begin{array}{l}\text { or belching } \\
\text { Globus }\end{array}$ & $0.11(0.05,0.19)$ & $0.86(0.79,0.92)$ & $0.40(0.21,0.61)$ & $0.55(0.47,0.62)$ & $0.82(0.39,1.74)$ & $1.03(0.93,1.14)$ \\
\hline
\end{tabular}

$\mathrm{PPV}$, positive predictive value; NPV, negative predictive value; LR+, positive likelihood ratio; LR -, negative likelihood ratio; GERD, gastroesophageal reflux disease; NCCP, non-cardiac chest pain.

Table 3. Factors Associated With Detection of Major Esophageal Motility Disorders Among Patients With Dysphagia $(\mathrm{n}=100)$

\begin{tabular}{|c|c|c|c|}
\hline Factors & Crude OR (95\% CI) & Adjusted OR (95\% CI) & $P$-value (LR-test) \\
\hline \multicolumn{4}{|l|}{ Demographic characteristics } \\
\hline Gender: female vs male & $0.59(0.26,1.35)$ & $0.93(0.30,2.87)$ & 0.904 \\
\hline Age group (ref. $\geq 65 \mathrm{yr}$ ) & & & $<0.001$ \\
\hline$<45 \mathrm{yr}$ & $1.36(0.35,5.27)$ & $1.22(0.27,5.50)$ & \\
\hline $45-65 \mathrm{yr}$ & $0.15(0.05,0.43)$ & $0.12(0.04,0.40)$ & \\
\hline \multicolumn{4}{|l|}{ Coexisting symptoms } \\
\hline GERD & $3.24(0.86,12.14)$ & $2.84(0.53,15.05)$ & 0.201 \\
\hline Chest pain/discomfort & $0.90(0.38,2.16)$ & $0.78(0.25,2.39)$ & 0.662 \\
\hline Nausea/vomiting & $4.55(0.97,21.44)$ & $4.45(0.72,27.35)$ & 0.086 \\
\hline Globus & $0.27(0.05,1.56)$ & $0.17(0.02,1.49)$ & 0.098 \\
\hline Belching & $1.84(0.35,9.64)$ & $1.27(0.19,8.71)$ & 0.803 \\
\hline Weight loss & $7.00(1.52,32.20)$ & $7.11(1.05,21.00)$ & 0.025 \\
\hline
\end{tabular}

LR, likelihood ratio; ref., reference group; GERD, gastroesophageal reflux disease. 
the relevant experienced personnel and high budgeting required for this technique. This study was conducted to evaluate the diagnostic yield of high-resolution esophageal manometry in correlation with esophageal symptoms.

First, we would like to emphasize that this investigation was conducted under the conditions of a referral center where all patients underwent basic investigations including esophagogastroduodenoscopy before esophageal manometries were performed. We did not routinely perform esophageal biopsies to rule out eosinophilic esophagitis because of very low prevalence of this condition in the Asian population (1 out of 5000 endoscopy examinations). ${ }^{10}$

Dysphagia was the most common indication for HREM (49.8\%) followed by GERD (17.4\%), which were mostly refractory symptoms in our study. We noticed an upward trend of refractory GERD as an indication or requirement for HREM in our population similar to the studies by Xiao et $\mathrm{al}^{11}$ (Western population) and Jain et $\mathrm{al}^{8}$ (Asian population). Besides these, we had a modest number of patients with globus symptoms that underwent HREM. Obviously, dysphagia revealed the highest yield of detecting an abnormal esophageal motility test (71.0\%); meanwhile, this yield was much lower in GERD (42.9\%) and globus patients (34.6\%). Major esophageal motility disorders were also more frequent in dysphagia patients (88.7\%), especially achalasia and EGJ outflow obstruction, which was a finding similar to those of other studies. This stood in contrast with nausea/vomiting or belching where minor esophageal motility disorders were the most predominant.

Interestingly, we found a significant proportion of major esophageal motility disorders, particularly EGJ outflow tract obstruction and jackhammer esophagus, in patients with non-cardiac chest pain, which differed from the findings of previous literature that have reported ineffective motility ${ }^{9}$ and achalasia ${ }^{12}$ to be common abnormal findings. Besides, major esophageal motility disorders may be found in non-dysphagia patients such as those with refractory GERD and globus symptoms.

Since the Chicago classification v3.0 simplified the classification of esophageal motor disorders compared to the former version (v2.0), several motor abnormalities shifted into the 'normal' category. In addition, the proportions of hypermotility and hypomotility disorders were also decreased. ${ }^{13}$ For patients with dysphagia, the likelihood of esophageal motor abnormality diagnosis is still highest for both classification versions. Moreover, Chicago classification v3.0 significantly identified esophageal motor disorders with higher symptom burden compared to $\mathrm{v} 2.0,{ }^{13}$ resulting in a larger impact on clinical application.

Because of the much higher clinical impact of major esophageal motility disorders, we attempted to evaluate the predictive ability of esophageal symptoms for major findings on HREM. The highest sensitivity for major esophageal motility disorders was observed in dysphagia (70.0\%). Despite showing the highest sensitivity, its positive predictive value was just $63.0 \%$, and its positive likelihood ratio (2.10) had a small effect. These findings emphasized the variety of esophageal symptoms and manometric findings. Furthermore, multiple logistic regression analysis demonstrated that the elderly patients with dysphagia who have coexisting weight loss were more likely to be diagnosed with the major abnormalities. In addition, although we noticed the high chances of endoscopic or surgical treatment in patients with dysphagia, these treatments were still needed in a minority group of non-dysphagia patients with major esophageal motility disorders.

Our findings indicated a higher yield of positive esophageal manometry findings among dysphagia patients, but this procedure was not a strongly beneficial test in patients with non-dysphagia to determine clinically relevant esophageal motor disorders. Therefore, we recommend the application of this testing strategy in clinical practice for Thailand and countries with limited resources. For refractory cases of non-dysphagia symptoms in which major motor esophageal disorders are suspected, HRM may be subsequently considered. However, apart from the diagnosis of esophageal motor disorders, HRM is recommended to accurately position the $\mathrm{pH}$ monitoring catheter in patients with reflux related conditions.

To the authors' knowledge, this is the first study to demonstrate the applicability of high-resolution esophageal manometry according to the latest Chicago classification in Southeast Asian countries, and it represents the real-life situation in a large tertiary care center. Moreover, we used the same machine and technique as the Chicago classification reference. However, this study has one limitation worth mentioning, the employment of a different procedure position-the semi-upright or supine positions instead of only the supine position. Nevertheless, the motility patterns may differ between liquid and solid boluses, especially in cases of swallowing-related symptoms.

In conclusion, high-resolution esophageal manometry yields a higher diagnostic value in patients with dysphagia; therefore, manometry should be performed routinely after the exclusion of organic esophageal diseases. Manometry is not a strongly beneficial diagnostic test in patients with non-dysphagia except where major esophageal motor disorders are suspected.

Acknowledgements: The authors are grateful to the Excellence Center, Nanthana-Kriangkrai Chotiwattanaphan Institute of Gas- 
troenterology and Hepatology, of Songklanagarind Hospital for providing the HRM facilities.

\section{Financial support: None.}

\section{Conflicts of interest: None.}

Author contributions: Sawangpong Jandee made a substantial contribution to the study concept, accessing of data, drafting of the manuscript, and critical revision of the manuscript; and Kasemsak Jandee was responsible for analysis, interpretation of data, and revision of the manuscript.

\section{References}

1. Pandolfino JE, Kahrilas PJ. AGA technical review on the clinical use of esophageal manometry. Gastroenterology 2005;128:209-224.

2. Van Hoeij FB, Bredenoord AJ. Clinical application of esophageal highresolution manometry in the diagnosis of esophageal motility disorder. $\mathrm{J}$ Neurogastroenterol Motil 2016;22:6-13.

3. Carlson DA, Pandolfino JE. High-resolution manometry in clinical practice. Gastroenterol Hepatol 2015;11:374-384.

4. Carlson DA, Kahliras PJ. How to effectively use high-resolution esophageal manometry. Gastroenterology 2016;151:789-792.

5. Rohof WOA, Bredenoord AJ. Chicago classification of esophageal mo- tility disorders: lessons learned. Curr Gastroenterol Rep 2017;19:37.

6. Yadlapati R, Gawron AJ, Keswani RN, et al. Identification of quality measures for performance of and interpretation of data from esophageal manometry. Clin Gastroenterol Hepatol 2016;14:526-534, e1.

7. Kahrilas PJ, Bredenoord AJ, Fox M, et al. The Chicago classification of esophageal motility disorders, v3.0. Neurogastroenterol Motil 2015;27:160-174.

8. Jain M, Srinivas M, Bawane P, Venkataraman J. Does Chicago classification address symptom correlation with high-resolution esophageal manometry? Euroasian J Hepatogastroenterol 2017;7:122-125.

9. Goyal O, Bansal M, Sood A. Esophageal motility disorders-symptomatic and manometric spectrum in Punjab, northern India. Indian J Gastroenterol 2017;36:202-208.

10. Kinoshita Y, Ishimura N, Oshima N, Ishihara S. Systematic review: eosinophilic esophagitis in Asian countries. World J Gastroenterol 2015;21:8433-8440

11. Xiao Y, Kahrilas PJ, Nicodème F, Lin Z, Roman S, Pandolfino JE. Lack of correlation between HRM metrics and symptoms during the manometric protocol. Am J Gastroenterol 2014;109:521-526.

12. Bansal RK, Nasa M, Patil GK, et al. Spectrum of esophageal motility disorders in patients with motor dysphagia and noncardiac chest pain-a single center experience. J Dig Endosc 2017;8:68-71.

13. Patel A, Cassell B, Sainani N, et al. Comparison of motor diagnoses by Chicago classification versions 2.0 and 3.0 on esophageal high-resolution manometry. Neurogastroenterol Motil 2017;29:e13042. 